\title{
The perils of using registry data to compare the survival and cost of radical cystectomy and trimodality therapy in bladder cancer
}

\author{
Abhishek A. Solanki ${ }^{1}$, Stanley L. Liauw ${ }^{2}$ \\ ${ }^{1}$ Department of Radiation Oncology, Stritch School of Medicine, Loyola University Chicago, Chicago, IL, USA; ${ }^{2}$ Department of Radiation and \\ Cellular Oncology, Pritzker School of Medicine, University of Chicago, Chicago, IL, USA \\ Correspondence to: Abhishek A. Solanki, MD, MS. Department of Radiation Oncology, Loyola University Medical Center, 2160 S. First Ave, \\ Maywood, IL 60153, USA. Email: Abhishek.Solanki@lumc.edu. \\ Provenance: This is an invited article commissioned by the Section Editor Dr. Xiao Li (Department of Urology, Jiangsu Cancer Hospital \& Jiangsu \\ Institute of Cancer Research \& Affiliated Cancer Hospital of Nanjing Medical University, Nanjing, China). \\ Comment on: Williams SB, Shan Y, Ray-Zack MD, et al. Comparison of Costs of Radical Cystectomy vs Trimodal Therapy for Patients With \\ Localized Muscle-Invasive Bladder Cancer. JAMA Surg 2019;154:e191629.
}

Submitted Nov 01, 2019. Accepted for publication Dec 20, 2019.

doi: $10.21037 /$ tau. 2019.12 .35

View this article at: http://dx.doi.org/10.21037/tau.2019.12.35

Radical Cystectomy and trimodality therapy with maximal transurethral resection of bladder tumor followed by concurrent chemoradiotherapy are standard curative treatment options for patients with muscle-invasive bladder cancer (MIBC). There are no robust randomized data available to determine the comparative effectiveness between these treatment modalities. Both treatment approaches are supported by National Comprehensive Cancer Network (NCCN) and American Urological Association guidelines $(1,2)$. However, fundamental differences in the two therapeutic approaches and associated risks create a divergent choice for clinicians and patients, which can introduce some complexity in decision making.

One important factor to consider is the cost to the patient and healthcare system. In the August 2019 edition of $7 A M A$ Surgery, Dr. Williams and his team performed a population-based retrospective cohort study of patients with MIBC treated with either radical cystectomy or trimodality therapy in the Surveillance, Epidemiology, and End Results-Medicare (SEER-Medicare) database to compare total medicare costs within the first year of diagnosis (3). After performing an analysis using inverse probability of treatment-weighted propensity score models, the investigators concluded that trimodality therapy was associated with worse cause-specific and overall survival and higher costs than radical cystectomy.

We commend the study team for attempting to investigate differences between the treatments outside of direct oncologic efficacy and toxicity, but there are significant limitations to the study design and the study methodology that raise concern that confounding could have impacted the findings for both survival outcomes and cost. Therefore, the results should be interpreted with caution.

The primary limitation of this study is the observational study design. Only randomization can control for known and unknown confounders and truly establish causality, and observational studies can only determine correlation. One randomized trial has been attempted to compare radical cystectomy with radiation therapy-the Selective bladder Preservation Against Radical Excision (SPARE) trial (4). In this study, patients with T2-3 N0M0 urothelial carcinoma of the bladder underwent 3 cycles of gemcitabine/cisplatin chemotherapy, followed by cystoscopy. Patients who had $\geq \mathrm{pT} 2$ disease proceeded to radical cystectomy, while those with $\leq \mathrm{pT} 1$ disease received one more cycle of chemotherapy and then were randomized to either radical cystectomy or radiation therapy. However, due to poor accrual and low compliance with randomized treatment, only 45 patients were enrolled, and the independent trial steering committee recommended closing the study during the feasibility phase. Although patients in the radical cystectomy group had improved 2-year loco-regional recurrence rates, they had higher grade 3-4 CTCAE toxicity and no difference in 
Table 1 Observational studies comparing survival outcomes with radical cystectomy (RC) and trimodality therapy (TMT)

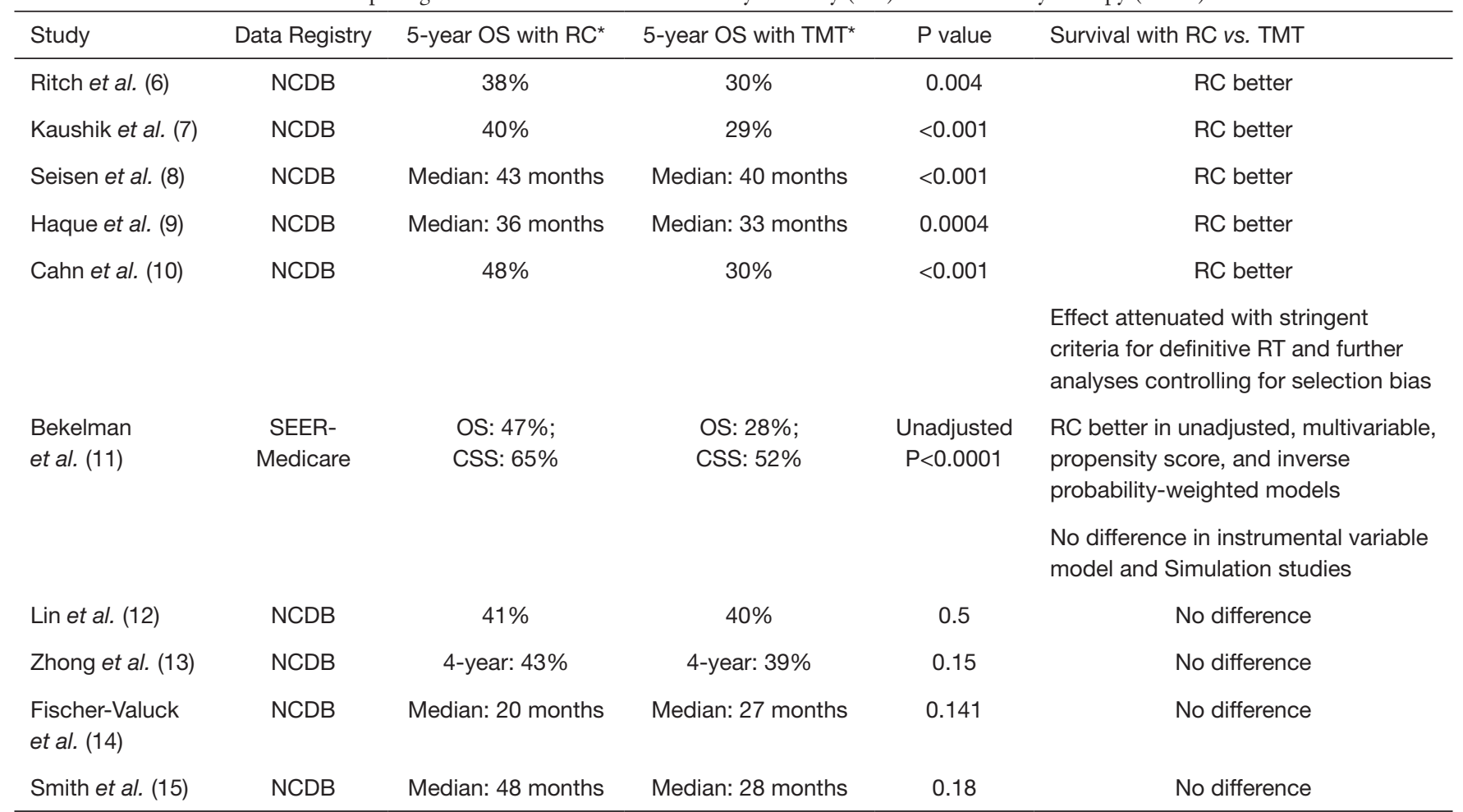

*, unless specified below. OS, overall survival; NCDB, National Cancer Database; SEER, Surveillance, Epidemiology, and End Results; CSS, cause-specific survival; RT, radiation therapy.

metastasis-free survival or disease-specific survival between groups (4). Only 2 of the 20 patients in the radiation therapy group received concurrent chemotherapy, and therefore comparisons between trimodality therapy and radical cystectomy are not possible from this study. No other randomized trials are ongoing, and thus there are unlikely to be randomized data to help guide selection of these modalities soon.

The availability and "real world" nature of populationbased registries make them a promising resource for comparative effectiveness research in the absence of randomized data. However, when comparing surgery $v s$. radiotherapy, observational studies should not be used to make conclusions about survival differences due to the impact of selection bias on outcomes; selection bias is a critical confounder. There is no way to control for surgical candidacy. That is, patients who receive radiation therapy include those who may have not been candidates for surgery due to comorbidity and advanced disease-a group with a significantly worse prognosis. This can lead to bias towards worse outcomes with radiation therapy. In a recent analysis, 350 studies comparing two treatments using an observational registry (SEER, SEER-Medicare, or the National Cancer Database) were matched to 121 randomized trials comparing the same two treatments (5). Whereas $68 \%$ of the observational comparisons reported a statistically significant survival difference, only $31 \%$ of randomized trials reported significant survival differences. Only $40 \%$ of matched studies reported the same conclusion; there was no correlation greater than chance alone between the results of the observational studies and the results of the randomized trials.

Indeed, there have been at least 10 comparative effectiveness studies comparing radical cystectomy and bladder preserving therapy using SEER-Medicare and the National Cancer Database, with five studies showing better survival associated with radical cystectomy and five showing no difference when controlling for potential confounders (Table 1) (6-15). Bekelman et al. specifically attempted to determine whether analytic methods can impact the results of this comparison using population-based datasets and found that multivariable and propensity score analyses 
favored improved survival with radical cystectomy, while instrumental variable or simulation study designs concluded no difference (11). Based on these findings, the results of observational comparisons of radical cystectomy and trimodality therapy are suspect.

There are multiple features of this study's specific methodology that may threaten the internal validity of the conclusions. Most importantly, the definition of the radiation therapy component of trimodality therapy was problematic. A standard course of curative radiation therapy for MIBC in the U.S. is $\sim 32-36$ fractions. Although there are hypofractionated regimens using as low as 20 fractions, in the U.S. there is very little use of these fractionations in the curative setting (16). There is no way of knowing what percent of patients actually received curative radiation therapy doses. Only $23.9 \%$ of patients in the trimodality therapy group had the number of radiation therapy fractions known, and in this minority with known number of fractions, the median number of fractions was 27 and interquartile range was $12-37$. The authors found that there was compromised survival in the patients receiving $<27$ fractions, consistent with the difference between palliative and curative treatment. Therefore, the trimodality therapy group likely included a substantial proportion of patients who were receiving radiotherapy for palliation-a clinical situation that would be associated with inferior survival and greater overall costs due to the advanced nature of disease, compared with radical cystectomy or curative trimodality therapy.

Similarly, the codes used to define the radiation therapy group included codes that are not part of standard curative radiation therapy for MIBC: superficial and orthovoltage radiation therapy (ICD procedure code: 92.21-2) stereotactic radiosurgery (SRS; 92.30-39), radioisotope therapy (92.20) intraoperative radiotherapy (IORT; 92.4 and 92.41), and brachytherapy (92.27). Additionally, these modalities can be more expensive than the standard fractionated external beam RT used in this setting. This suggests either aberrant practice in the patients included in this study or mis-coding of data-both of which reduce the internal validity of the cost and survival results.

In addition to the inclusion of non-standard and noncurative radiation therapy in the trimodality groups, the use of six months after TURBT as the window for administration of curative trimodality therapy is also problematic and may have confounded the results. Six months is a large window after TURBT for administration of trimodality therapy. This would allow inclusions of patients with competing medical issues that may have led to a delay in delivery of curative treatment, worsened survival, and increased cost.

In the absence of randomized data, and with the limited insights one can gain from population-based observational comparative-effectiveness studies, the question of whether radical cystectomy or trimodality therapy are more efficacious still remains unanswered. But, perhaps this is not the right question to ask. The drastically different logistics of both approaches and the impact on quality of life compel clinicians and patients to choose one modality over the other via shared decision-making (17).

One potentially valuable avenue to explore is the ability of biomarkers that can help guide selection of treatment modality. Efstathiou et al. performed transcriptional profiling of tumors from patients with MIBC and found that in patients undergoing trimodality therapy, decreased tumor immune infiltration was associated with worse diseasespecific survival, while increased tumor stromal infiltration was associated with worse disease-specific survival in the setting of radical cystectomy with or without neoadjuvant chemotherapy (18). Several ongoing studies are evaluating the incorporation of biomarkers into treatment selection. For example, Alliance A031701 (NCT03609216) is studying the use of DNA damage response gene alterations to guide local therapy in the setting of neoadjuvant chemotherapy, although in this study, patients with DNA damage response gene alterations and $<\mathrm{cT} 1$ disease after neoadjuvant chemotherapy undergo no local therapy, while all others undergoing radical cystectomy or trimodality therapy.

Furthermore, up to $30-50 \%$ of patients with MIBC will develop disease recurrence after radical cystectomy or trimodality therapy $(19,20)$. Perhaps it is more realistic and practical to improve on the platforms of radical cystectomy and trimodality therapy to improve survival. Multiple trials are determining the role of neoadjuvant and adjuvant checkpoint immunotherapy after RC (NCT03244384; NCT03924856; NCT03674424; NCT03234153; NCT03732677). For patients undergoing trimodality therapy, SWOG/NRG 1806 (NCT03775265) is studying the role of concurrent and adjuvant atezolizumab in nodenegative MIBC, and CCTG BL13 (NCT03768570) is studying adjuvant durvalumab after trimodality therapy in this setting. ECOG/NRG 8185 is under development and will determine if concurrent and adjuvant durvalumab improves complete clinical response rates with trimodality therapy in node-positive MIBC.

In conclusion, registry-based and population-based 
observational comparative-effectiveness studies are prone to selection bias confounding the impact of treatment modality on survival outcomes. For MIBC, radical cystectomy and trimodality therapy are two evidence based, guidelinesupported curative modalities, and observational studies are unlikely to provide practice-changing data comparing differential outcomes between these approaches. In the absence of randomized data, clinicians should conduct shared decision making with patients with equipoise regarding disease outcome, while considering a patient's relative candidacy, as well as individual preferences, for either therapy.

\section{Acknowledgments}

None.

\section{Footnote}

Conflicts of Interest: The authors have no conflicts of interest to declare.

Ethical Statement: The authors are accountable for all aspects of the work in ensuring that questions related to the accuracy or integrity of any part of the work are appropriately investigated and resolved.

\section{References}

1. Flaig TW. NCCN Guidelines Updates: Management of Muscle-Invasive Bladder Cancer. J Natl Compr Canc Netw 2019;17:591-3.

2. Chang SS, Bochner BH, Chou R, et al. Treatment of Non-Metastatic Muscle-Invasive Bladder Cancer: AUA/ ASCO/ASTRO/SUO Guideline. J Urol 2017;198:552-9.

3. Williams SB, Shan Y, Ray-Zack MD, et al. Comparison of Costs of Radical Cystectomy vs Trimodal Therapy for Patients With Localized Muscle-Invasive Bladder Cancer. JAMA Surg 2019;154:e191629.

4. Huddart RA, Birtle A, Maynard L, et al. Clinical and patient-reported outcomes of SPARE - a randomised feasibility study of selective bladder preservation versus radical cystectomy. BJU Int 2017;120:639-50.

5. Soni PD, Hartman HE, Dess RT, et al. Comparison of Population-Based Observational Studies With Randomized Trials in Oncology. J Clin Oncol 2019;37:1209-16.

6. Ritch CR, Balise R, Prakash NS, et al. Propensity matched comparative analysis of survival following chemoradiation or radical cystectomy for muscle-invasive bladder cancer. BJU Int 2018;121:745-51.

7. Kaushik D, Wang H, Michalek J, et al. Chemoradiation Vs Radical Cystectomy for Muscle-invasive Bladder Cancer: A Propensity Score-weighted Comparative Analysis Using the National Cancer Database. Urology 2019;133:164-74.

8. Seisen T, Sun M, Lipsitz SR, et al. Comparative Effectiveness of Trimodal Therapy Versus Radical Cystectomy for Localized Muscle-invasive Urothelial Carcinoma of the Bladder. Eur Urol 2017;72:483-7.

9. Haque $W$, Verma V, Butler EB, et al. Radical Cystectomy Versus Chemoradiation for Muscle-invasive Bladder Cancer: Impact of Treatment Facility and Sociodemographics. Anticancer Res 2017;37:5603-8.

10. Cahn DB, Handorf EA, Ghiraldi EM, et al. Contemporary use trends and survival outcomes in patients undergoing radical cystectomy or bladderpreservation therapy for muscle-invasive bladder cancer. Cancer 2017;123:4337-45.

11. Bekelman JE, Handorf EA, Guzzo T, et al. Radical cystectomy versus bladder-preserving therapy for muscleinvasive urothelial carcinoma: examining confounding and misclassification biasin cancer observational comparative effectiveness research. Value Health 2013;16:610-8.

12. Lin HY, Ye H, Kernen KM, et al. National Cancer Database Comparison of Radical Cystectomy vs Chemoradiotherapy for Muscle-Invasive Bladder Cancer: Implications of Using Clinical vs Pathologic Staging. Cancer Med 2018;7:5370-81.

13. Zhong J, Switchenko J, Jegadeesh N, et al. Comparison of Outcomes in Patients With Muscle-Invasive Bladder Cancer Treated With Radical Cystectomy Versus BladderPreserving Chemoradiation. Int J Radiat Oncol Biol Phys 2016;96:S93-4.

14. Fischer-Valuck BW, Rao YJ, Rudra S, et al. Treatment Patterns and Overall Survival Outcomes of Octogenarians with Muscle Invasive Cancer of the Bladder: An Analysis of the National Cancer Database. J Urol 2018;199:416-23.

15. Smith AB, Deal AM, Woods ME, et al. Muscle-invasive bladder cancer: evaluating treatment and survival in the National Cancer Data Base. BJU Int 2014;114:719-26.

16. Solanki AA, Martin B, Korpics M, et al. BladderPreserving Therapy Patterns of Care: A Survey of US Radiation Oncologists. Int J Radiat Oncol Biol Phys 2017;99:383-7. 
17. Mak KS, Smith AB, Eidelman A, et al. Quality of Life in Long-term Survivors of Muscle-Invasive Bladder Cancer. Int J Radiat Oncol Biol Phys 2016;96:1028-36.

18. Efstathiou JA, Mouw KW, Gibb EA, et al. Impact of Immune and Stromal Infiltration on Outcomes Following Bladder-Sparing Trimodality Therapy for Muscle-Invasive Bladder Cancer. Eur Urol 2019;76:59-68.

19. Parekh DJ, Reis IM, Castle EP, et al. Robot-assisted

Cite this article as: Solanki AA, Liauw SL. The perils of using registry data to compare the survival and cost of radical cystectomy and trimodality therapy in bladder cancer. Transl Androl Urol 2019;8(Suppl 5):S533-S537. doi: 10.21037/ tau.2019.12.35 radical cystectomy versus open radical cystectomy in patients with bladder cancer (RAZOR): an openlabel, randomised, phase 3, non-inferiority trial. Lancet 2018;391:2525-36.

20. James ND, Hussain SA, Hall E, et al. Radiotherapy with or without Chemotherapy in Muscle-Invasive Bladder Cancer. N Engl J Med 2012;366:1477-88. 